\title{
Alimentação e padrões ecomorfológicos das espécies de peixes de riacho do alto rio Tocantins, Goiás, Brasil
}

\author{
Rosana Mazzoni ${ }^{1}$, Maíra Moraes ${ }^{1,2}$, Carla F. Rezende ${ }^{3}$ \& Jean Carlos Miranda ${ }^{1}$
}

1. Laboratório de Ecologia de Peixes, Departamento de Ecologia, Universidade do Estado do Rio de Janeiro, Av. São Francisco Xavier 525, 20550-010 Rio de Janeiro, RJ, Brasil. (mazzoni@uerj.br)

2. Programa de Pós-Graduação em Ecologia e Evolução, Departamento de Ecologia, Instituto de Biologia, Universidade do Estado do Rio de Janeiro.

3. Departamento de Biologia, Centro de Ciências, Universidade Federal do Ceará, Campus do Pici, 60455-970 Fortaleza, CE, Brasil.

\begin{abstract}
Feeding and ecomorphological patterns of stream-dwelling fishes from the upper Tocantins river, Goiás, Brazil. In the present study we aimed to compare ecomorphological and feeding data of 18 stream-dwelling fish species from the upper Tocantins river, in order to test if morphological patterns confirm the trophic ones and if they could be used as predictive characters in the analysis of the trophic structure of this community. Feeding analysis revealed a wide range of food items consumed by the fishes and allowed the recognition of three major trophic groups: carnivorous/insectivorous/piscivorous, omnivorous and detritivorous/herbivorous. Principal Component Analysis, based on the morphometric indices, organized the studied species according to head size, eye diameter, mouth position and body depth and allowed the recognition of two major ecomorphological groups. We concluded that the ecomorphological analysis efficiently described specific ecological characters, reflected feeding and habitat use preferences and could be used as trophic structure predictors of the studied community.
\end{abstract}

KEYWORDS. Trophic ecology, stream-dwelling fishes, Amazon basin, allochthonous resources, autochthonous resources.

\begin{abstract}
RESUMO. No presente trabalho, comparamos a morfologia e o hábito alimentar de 18 espécies de peixes do alto rio Tocantins a fim de verificar se os padrões morfológicos obtidos para o conjunto das espécies estudadas corroboram os padrões tróficos e, portanto, se podem ser usados como caracteres preditivos da estrutura trófica da comunidade. A análise da dieta revelou ampla variedade de itens consumidos e distinção de três grandes grupos tróficos: espécies carnívoras/insetívoras/piscívoras, onívoras e herbívoras. A Análise dos Componentes Principais, baseada nos índices morfológicos, determinou a ordenação das espécies de acordo com tamanho da cabeça, diâmetro do olho, posição da boca e altura do corpo e permitiu a distinção de dois grandes grupos de espécies. Os grupos obtidos a partir dos índices morfológicos corroboram o padrão obtido a partir da análise da dieta das espécies. A análise ecomorfológica revelou-se uma ferramenta adequada na descrição de aspectos ecológicos das espécies estudadas, refletindo preferências alimentares e de uso do hábitat e pode ser usada como método preditivo da estrutura trófica da comunidade estudada.
\end{abstract}

PALAVRAS-CHAVE. Ecologia trófica, peixes de riachos, bacia Amazônica, recursos alóctones, recursos autóctones.

A relação entre morfologia e comportamento ecológico tem sido investigada em populações de diversos sistemas fluviais e comunidades de peixes (e.g. Felley, 1984; Mahon, 1984; Watson \& BaLON, 1984; Wikramanayake, 1990; Douglas \& Matthews, 1992; Winemiller et al., 1995; STEVEns \& WiLLig, 2000; TeIXeIRA \& BenNemann, 2007) e vários estudos permitiram a descrição de padrões consistentes para a relação entre a morfologia e os atributos ecológicos das espécies (i.e. XIE et al., 2001). Está amplamente demonstrado e discutido na literatura que a morfologia, o uso dos recursos tróficos e a forma de exploração dos demais recursos ambientais são parâmetros correlacionados (WATSON \& BALON, 1984; BALON et al., 1986; BARRELla et al., 1994; BEAUMORD \& Petrere-JR, 1994; Winemiller et al., 1995; Clifton \& Motta, 1998; Hugueny \& Pouilly, 1999; Pouilly et al., 2003) e, nesse contexto, a análise ecomorfológica é uma importante ferramenta para a descrição de padrões de estrutura de comunidades de peixes (WiKRAMANAYAKE, 1990).

Diversos trabalhos sobre peixes de riachos da região temperada têm demonstrado a relação entre a morfologia e o hábito alimentar e suas consequências para o uso e ocupação do hábitat gerando, assim, modelos preditivos da estrutura trófica de comunidades naturais, a partir dos padrões morfológicos exibidos pelas diferentes espécies (e.g. Gatz, 1979; Wikramanayake, 1990; Winemiller et al., 1995; Piet, 1998; Hugueny \& Pouilly, 1999; XIE et al., 2001). Por outro lado, dada a escassez de estudos dessa natureza para peixes tropicais, esse tipo de abordagem ainda não é possível (Pouilly $e t$ al., 2003). Por ser um dos grupos de maior diversidade morfológica e ecológica entre os vertebrados (FreIRE \& Agostinho, 2001), os peixes constituem importante fonte de respostas para se inferir sobre as relações entre a morfologia, a ecologia (Cunico \& AgostinHo, 2006) e o uso do hábitat (RINCón, 1999).

Neste estudo comparamos a morfologia relacionada ao comportamento trófico e o hábito alimentar de 18 espécies de peixes do alto rio Tocantins, a fim de verificar se os padrões morfológicos corroboram os padrões tróficos e, portanto, se podem ser usados como caracteres preditivos da estrutura trófica da comunidade estudada. Além disso, discutimos possíveis casos de convergência e/ou divergência morfológica para espécies relacionadas e não relacionadas filogeneticamente.

\section{MATERIAL E MÉTODOS}

Área de estudo e amostragem. O presente estudo foi desenvolvido no córrego Cavalo, um riacho de $3^{\text {a }}$ 
ordem do alto Tocantins ( $\left.14^{\circ} 26^{\prime} 12^{\prime \prime} \mathrm{S}, 48^{\circ} 34^{\prime} 53^{\prime \prime} \mathrm{W}\right)$. O trecho estudado é caracterizado por zonas intercaladas de rápidos com fundo rochoso e pedregoso, corredeiras e remansos de fundo areno-lodoso. A vegetação marginal é escassa e composta por gramíneas e plantas aquáticas de pequeno porte, parcial ou completamente submersas. A cobertura vegetal é quase total e é representada por árvores de grande porte que formam o dossel paralelo às margens da área de estudo. Os peixes utilizados no presente estudo foram obtidos por pesca elétrica (corrente alternada-1600 W, $220 \mathrm{~V}, 2-4 \mathrm{~A}$; MAZzoni et al., 2000) durante os meses de seca (abril e junho de 1996) e chuvas (dezembro de 1996 e janeiro de 1997). A cada ocasião de coleta eram obtidos aproximadamente 15 exemplares, que eram fixados em formalina $10 \%$ e, posteriormente, conservados em álcool 70\%. Exemplares testemunhos das espécies estudadas foram depositados na coleção ictiológica do Museu Nacional do Rio de Janeiro (MNRJ 36136-36178).

Análise da dieta e ecomorfologia. De cada exemplar coletado foi retirado, de acordo com a espécie, o estômago ou o primeiro terço do intestino para análise do hábito alimentar. A dieta das espécies estudadas foi determinada através da análise dos conteúdos estomacais e/ou intestinais a partir do método volumétrico (Vo) (Hyslop, 1980). Cada item alimentar registrado foi identificado até o menor nível taxonômico possível com o auxílio de bibliografia especializada (MERRIT \& CuMMINS, 1984; PÉrez, 1988) e, posteriormente, agrupado em categorias ecológicas mais amplas: insetos alóctones, insetos autóctones, sementes, algas, fragmento vegetal e peixes.

Para cada exemplar coletado foram registradas sete medidas morfológicas associadas à alimentação, sendo seis relativas à morfologia externa (Fig. 1) e uma relativa ao comprimento do intestino distendido. Essas medidas são as mesmas propostas por GATZ (1979). A partir destas medidas foram obtidos seis índices morfológicos apresentados e discutidos em WATSON \& BALON (1984) e Hugueny \& Pouilly (1999): (1) diâmetro relativo do olho (DRO): representa a relação diâmetro do olho e o comprimento da cabeça e é um atributo diretamente relacionado com a importância da visão na alimentação, podendo também indicar a posição vertical na coluna d'água, pois espécies que habitam águas mais profundas tendem a ter olhos menores (WIKRAMANAYAKE, 1990); (2) altura relativa do corpo (ARC): representa a relação entre a altura do corpo e o comprimento padrão e retrata o grau de achatamento do corpo, é um índice que apresenta relação inversa com o uso de ambientes de corredeira; (3) comprimento relativo da cabeça (CRC): representa a relação entre o comprimento da cabeça e o comprimento padrão, sendo que seus altos valores sugerem espécies que se alimentam de itens relativamente grandes; (4) altura relativa da boca (ARB): representa a relação entre a altura da boca e o comprimento padrão e é um atributo também relacionado com o tamanho relativo dos itens alimentares, assim como o tamanho da cabeça; (5) comprimento relativo do intestino (CRI): representa a relação entre o comprimento do intestino distendido e o comprimento padrão; (6) orientação da boca (OB): representa a posição da boca em relação à ponta do focinho e pode ser terminal, superior e ventral.

Análise dos dados. A fim de determinar a existência de diferentes padrões morfológicos entre as diferentes espécies estudadas realizou-se uma Análise de Componentes Principais (PCA - PC-Ord v. 4.0; MCCUNE \& MEFFord, 1997) com base nos valores médios dos índices morfológicos de cada espécie (Tab. I). Os eixos retidos para interpretação dos resultados foram aqueles cujos autovalores obtidos foram superiores aos autovalores de broken-stick (MCCunE \& MEFFORD, 1997). Da mesma forma, a detecção de grupos de espécies com semelhança no uso dos recursos tróficos foi realizada mediante Análise de Agrupamento (índice de Bray-Curtis, distância UPGMA - Pcord v. 4.0; McCune \& MefFord, 1997) baseada nas diferentes categorias alimentares registradas na análise da dieta. Utilizou-se a aproximação assintótica de Mantel baseada na distância de Sorensen (Bray-Curtis - Pcord v. 4.0; Douglas \& EndLeR, 1982) para avaliar a co-variância entre as matrizes ecomorfológica e trófica. A fim de testar o efeito da filogenia na correlação com a dieta e a morfologia (Douglas \& Matthews, 1992; Casatti \& Castro, 2006),
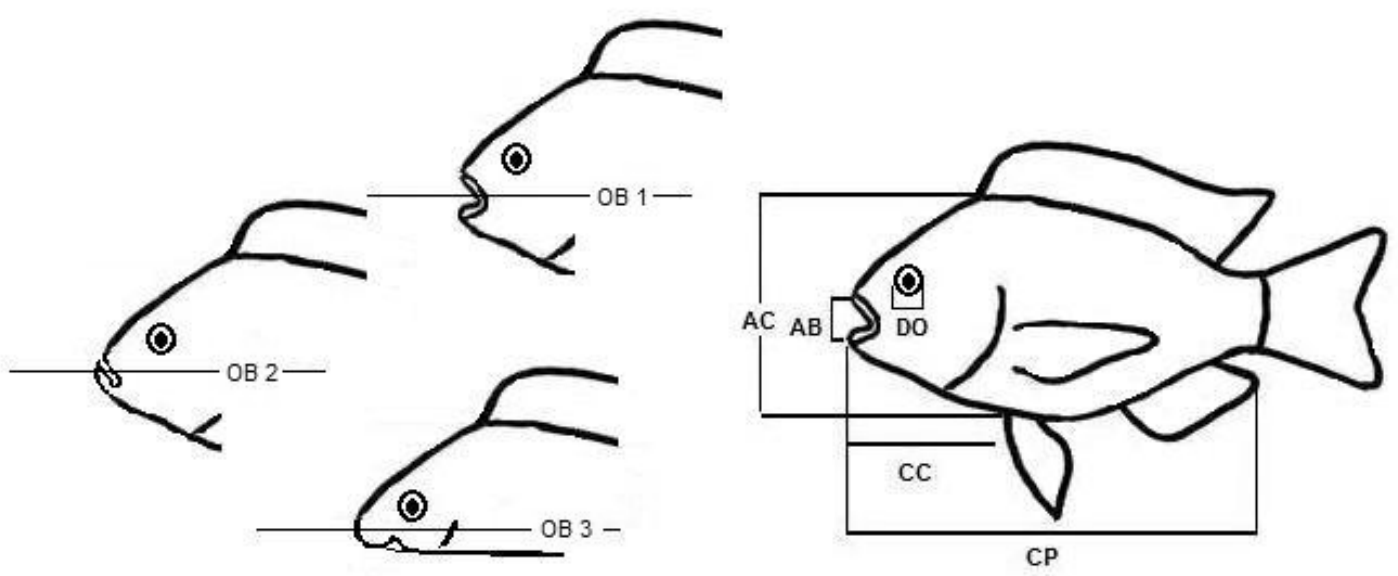

Figura 1. Variáveis morfológicas registradas para cada exemplar estudado (AB, abertura da boca - maior distância entre os lábios; AC, altura do corpo - maior distância ventro-dorsal perpendicular ao eixo longitudinal; CC, comprimento da cabeça - distância entre o focinho e o opérculo; CP, comprimento padrão - distância entre a ponta do focinho e o final do pedúnculo caudal; DO, diâmetro do olho; OB, orientação da boca: 1, terminal; 2, superior; 3, ventral). 
procedeu-se um teste de Mantel (Pcord v. 4.0; Douglas \& ENDLER, 1982). Para tal, foram utilizadas as mesmas matrizes ecomorfológica e trófica e comparamos com uma matriz de distância taxonômica construída segundo Douglas \& MATTHEws (1992), na qual o valor 1 é atribuído a pares de espécies congenéricas, o valor 2 é atribuído a pares de espécies de gêneros diferentes mas pertencente a mesma família, o valor 3 é atribuído a pares de espécies pertencentes a diferentes famílias porém da mesma ordem e o valor 4 é atribuído a pares de espécies pertencentes a diferentes ordens.

\section{RESULTADOS}

Foram analisados exemplares pertencentes a dezoito espécies (Tab. I). A análise da dieta das espécies indicou ampla variedade de itens consumidos, os quais compuseram sete categorias ecológicas mais amplas: (1) insetos alóctones, que reuniram Hymenoptera, Coleóptera e Megaloptera; (2) insetos autóctones, que reuniram larvas de Megaloptera, Plecoptera, Trichoptera, Chironomidae, Ephemeroptera, Simullidae, Lepidoptera, Odonata e Coleoptera; (3) sementes; (4) sedimento, que reuniu matéria orgânica em decomposição, areia e lodo; (5) algas, que reuniram diatomáceas, cianobactérias, clorofíceas e algas filamentosas; (6) fragmento vegetal e (7) peixes (Tab. II). Com base no volume consumido de cada categoria ecológica agrupamos as dezoito espécies em três guildas tróficas (carnívoros/insetívoros e/ou piscívoros, onívoros e detritívoros/herbívoros; Tab. II). A análise de agrupamento (Fig. 2), baseada nessas dezoito espécies, corroborou a distinção das guildas tróficas (Tab. II). As espécies carnívoras/insetívoras e/ou piscívoras foram reunidas no grupo I (gr.1), formado pelos Characiformes Astyanax sp., Creagrutus sp., $H$. aff. malabaricus, Knodus sp. 1, Knodus sp. 2. e Bryconops alburnoides Kner, 1858 e pelos Heptapteridae Imparfinis borodini Mees \& Cala, 1989, I. cf. schubarti e Phenacorhamdia sp. Essa classificação foi função da dieta baseada em insetos alóctones e autóctones e, apenas uma espécie (I. cf. schubarti) apresentou dieta também composta por peixes. As espécies onívoras foram reunidas no grupo II (gr.2), formado pelos Characiformes Apareiodon machrisi Travassos, 1957, Astyanax aff. bimaculatus, Leporinus ortomaculatus Garavello, 2000 e Characidium sp., e foram assim classificadas devido a presença na dieta de insetos, tanto alóctones quanto autóctones, e itens vegetais (sementes, algas e fragmentos vegetais). As espécies detritívoras/herbívoras compuseram o grupo III (gr.3), formado pelos Loricariidae Loricaria sp., Harttia punctata Rapp Py-Daniel \& Oliveira, 2001, Ancistrus minutus Fisch-Muller, Mazzoni \& Weber, 2001, Hypostomus plescostomus (Linnaeus, 1758) e Squaliforma emarginata (Valenciennes, 1840). Essa classificação se deu pela composição da dieta baseada em itens do sedimento (areia, lodo e material em

Tabela I. Valores médios (M) e respectivos desvios padrão (DP) dos índices morfológicos utilizados registrados para as dezoito espécies do córrego Cavalo, GO, Brasil (ARB, altura relativa da boca; ARC, altura relativa do corpo; CRC, comprimento relativo da cabeça; CRI, comprimento relativo do intestino; DRO, diâmetro relativo do olho; OB, orientação da boca: 1, terminal; 2, superior; 3, ventral).

\begin{tabular}{|c|c|c|c|c|c|c|c|c|c|c|c|}
\hline \multirow[t]{2}{*}{ Táxons } & \multicolumn{2}{|c|}{ DRO } & \multicolumn{2}{|c|}{ CRI } & \multicolumn{2}{|c|}{ ARC } & \multicolumn{2}{|c|}{$\mathrm{CRC}$} & \multicolumn{2}{|c|}{ ARB } & \multirow[t]{2}{*}{$\mathrm{OB}$} \\
\hline & M & $\mathrm{DP}$ & M & $\mathrm{DP}$ & $\mathrm{M}$ & $\mathrm{DP}$ & M & DP & $\mathrm{M}$ & DP & \\
\hline $\begin{array}{l}\text { CHARACIFORMES } \\
\text { CHARACIDAE }\end{array}$ & & & & & & & & & & & \\
\hline Astyanax aff. bimaculatus & 0,082 & 0,006 & 0,701 & 0,151 & 0,358 & 0,020 & 0,238 & 0,019 & 0,052 & 0,012 & 1 \\
\hline Astyanax sp. & 0,081 & 0,010 & 0,683 & 0,181 & 0,369 & 0,021 & 0,257 & 0,019 & 0,064 & 0,024 & 1 \\
\hline $\begin{array}{l}\text { Bryconops alburnoides } \\
\text { Kner, } 1858\end{array}$ & 0,098 & 0,011 & 0,678 & 0,182 & 0,361 & 0,057 & 0,237 & 0,018 & 0,083 & 0,021 & 1 \\
\hline Characidium sp. & 0,069 & 0,007 & 0,384 & 0,104 & 0,226 & 0,027 & 0,225 & 0,010 & 0,057 & 0,008 & 2 \\
\hline Creagrutus sp. & 0,086 & 0,011 & 0,691 & 0,188 & 0,277 & 0,019 & 0,232 & 0,013 & 0,063 & 0,008 & 1 \\
\hline Knodus sp. 1. & 0,093 & 0,016 & 0,673 & 0,144 & 0,296 & 0,024 & 0,237 & 0,018 & 0,071 & 0,026 & 1 \\
\hline $\begin{array}{l}\text { Knodus sp. } 2 . \\
\text { ANOSTOMIDAE }\end{array}$ & 0,105 & 0,010 & 0,734 & 0,055 & 0,335 & 0,009 & 0,252 & 0,016 & 0,063 & 0,008 & 1 \\
\hline $\begin{array}{l}\text { Leporinus ortomaculatus } \\
\text { Garavello, } 2000 \\
\text { ERYTHRINIDAE }\end{array}$ & 0,068 & 0,008 & 0,720 & 0,111 & 0,263 & 0,020 & 0,225 & 0,012 & 0,046 & 0,002 & 2 \\
\hline $\begin{array}{l}\text { Hoplias aff. malabaricus } \\
\text { PARODONTIDAE }\end{array}$ & 0,086 & 0,023 & 0,448 & 0,082 & 0,201 & 0,013 & 0,239 & 0,012 & 0,122 & 0,018 & 1 \\
\hline $\begin{array}{l}\text { Apareiodon machrisi } \\
\text { Travassos, } 1957 \\
\text { SILURIFORMES } \\
\text { LORICARIIDAE }\end{array}$ & 0,058 & 0,005 & 1,020 & 0,092 & 0,221 & 0,019 & 0,196 & 0,011 & 0,046 & 0,003 & 2 \\
\hline $\begin{array}{l}\text { Ancistrus minutus Fisch-Muller, } \\
\text { Mazzoni \& Weber, } 2001\end{array}$ & 0,049 & 0,003 & 10,433 & 1,724 & 0,173 & 0,019 & 0,376 & 0,042 & 0,096 & 0,012 & 3 \\
\hline $\begin{array}{l}\text { Harttia punctata Rapp } \\
\text { Py-Daniel \& Oliveira, } 2001\end{array}$ & 0,055 & 0,004 & 6,580 & 2,133 & 0,113 & 0,008 & 0,265 & 0,012 & 0,079 & 0,009 & 3 \\
\hline $\begin{array}{l}\text { Hypostomus plecostomus } \\
\text { (Linnaeus, 1758) }\end{array}$ & 0,065 & 0,007 & 17,075 & 6,426 & 0,209 & 0,017 & 0,346 & 0,016 & 0,089 & 0,016 & 3 \\
\hline $\begin{array}{l}\text { Squaliforma emarginata } \\
\text { (Valenciennes, 1840) }\end{array}$ & 0,059 & 0,005 & 10,684 & 2,639 & 0,172 & 0,010 & 0,344 & 0,008 & 0,081 & 0,006 & 3 \\
\hline $\begin{array}{l}\text { Loricaria sp. } \\
\text { HEPTAPTERIDAE }\end{array}$ & 0,032 & 0,004 & 6,966 & 1,289 & 0,095 & 0,009 & 0,227 & 0,018 & 0,061 & 0,005 & 3 \\
\hline $\begin{array}{l}\text { Imparfinis borodini } \\
\text { Mees \& Cala, } 1989\end{array}$ & 0,026 & 0,004 & 0,419 & 0,074 & 0,107 & 0,012 & 0,194 & 0,022 & 0,060 & 0,019 & 2 \\
\hline Imparfinis cf. schubartti & 0,041 & 0,004 & 0,425 & 0,115 & 0,165 & 0,019 & 0,199 & 0,013 & 0,082 & 0,010 & 2 \\
\hline Phenacorhamdia sp. & 0,018 & 0,004 & 0,381 & 0,120 & 0,113 & 0,016 & 0,179 & 0,015 & 0,057 & 0,018 & 2 \\
\hline
\end{tabular}


decomposição), além de itens de origem vegetal (algas, sementes e fragmentos vegetais).

Os valores médios e respectivos desvios padrão de cada índice morfológico são apresentados na tabela I. Dois eixos da PCA foram usados para interpretação por terem apresentado autovalores maiores que os autovalores do broken-stick (Tab. III). O primeiro componente (PC1) explicou 51,5\% da variância e o principal atributo desse componente foi a ARC e a OB, que caracteriza peixes de corpo alto e comprimido lateralmente e boca em posição terminal, discriminando as espécies de Characiformes, de hábito nectônico, daquelas de corpo relativamente mais baixo e, portanto, mais comprimidas dorso-ventralmente e boca em posição subterminal ou ventral, os Siluriformes, de hábito bentônico e epibentônico (Fig. 3).

O segundo componente da PCA (PC2) explicou $32,2 \%$ da variância, somando $83,8 \%$ de variância cumulativa nos dois primeiros eixos (Tab. III). Os principais atributos desse componente foram o CRC e o DRO, discriminando as espécies com cabeças mais longas e olhos grandes (Characidae, Erithrynidae e Loricariidae)

Tabela II. Relação das espécies de peixes estudadas no córrego Cavalo, GO, Brasil e respectivos valores médios do volume de cada item alimentar registrado na dieta das mesmas (Alg, algas; FrVe, fragmento vegetal; InAl, insetos alóctones; InAu, inseto autóctone; Peixe, peixes; Sed, sedimentos; Sem, semente).

\begin{tabular}{|c|c|c|c|c|c|c|c|}
\hline & InAl & InAu & Sem & Sed & Alg & $\mathrm{FrVe}$ & Peixe \\
\hline \multicolumn{8}{|c|}{ Carnívoros - Insetívoros e/ou Piscívoros } \\
\hline Astyanax sp. & 37,4 & 62,6 & & & & & \\
\hline Creagrutus sp. & 42,7 & 57,3 & & & & & \\
\hline Hoplias aff. malabaricus & 47,5 & 52,5 & & & & & \\
\hline Knodus sp. 1 & 56,2 & 43,8 & & & & & \\
\hline Knodus sp. 2 & 53,0 & 47,0 & & & & & \\
\hline Bryconops alburnoides & 58,8 & 41,2 & & & & & \\
\hline Imparfinis borodini & 99,0 & 1,0 & & & & & \\
\hline Imparfinis cf. schubarti & 59,6 & 0,7 & & & & & 39,7 \\
\hline Phenacorhamdia sp. & 99,8 & 0,2 & & & & & \\
\hline \multicolumn{8}{|c|}{ Onívoros } \\
\hline Apareiodon machrisi & 28,6 & 18,0 & 10,6 & 11,4 & 21,8 & 9,7 & \\
\hline Astyanax aff. bimaculatus & 19,6 & 25,2 & 14,0 & 11,8 & 19,6 & 9,8 & \\
\hline Leporinus ortomaculatus & 24,2 & 20,7 & & 11,4 & 31,1 & 12,6 & \\
\hline Characidium sp. & 39,8 & 24,9 & & 7,6 & 23,6 & 4,1 & \\
\hline \multicolumn{8}{|c|}{ Detritívoros/Herbívoros } \\
\hline Loricaria sp. & & & & 91,7 & 4,9 & 3,4 & \\
\hline Harttia punctata & & & & 71,1 & 25,2 & 3,7 & \\
\hline Ancistrus minutus & & & & 68,4 & 27,3 & 4,2 & \\
\hline Squaliforma emarginata & & & & 77,7 & 20,0 & 2,3 & \\
\hline Hypostomus plecostomus & & & & 77,1 & 14,9 & 8,1 & \\
\hline
\end{tabular}

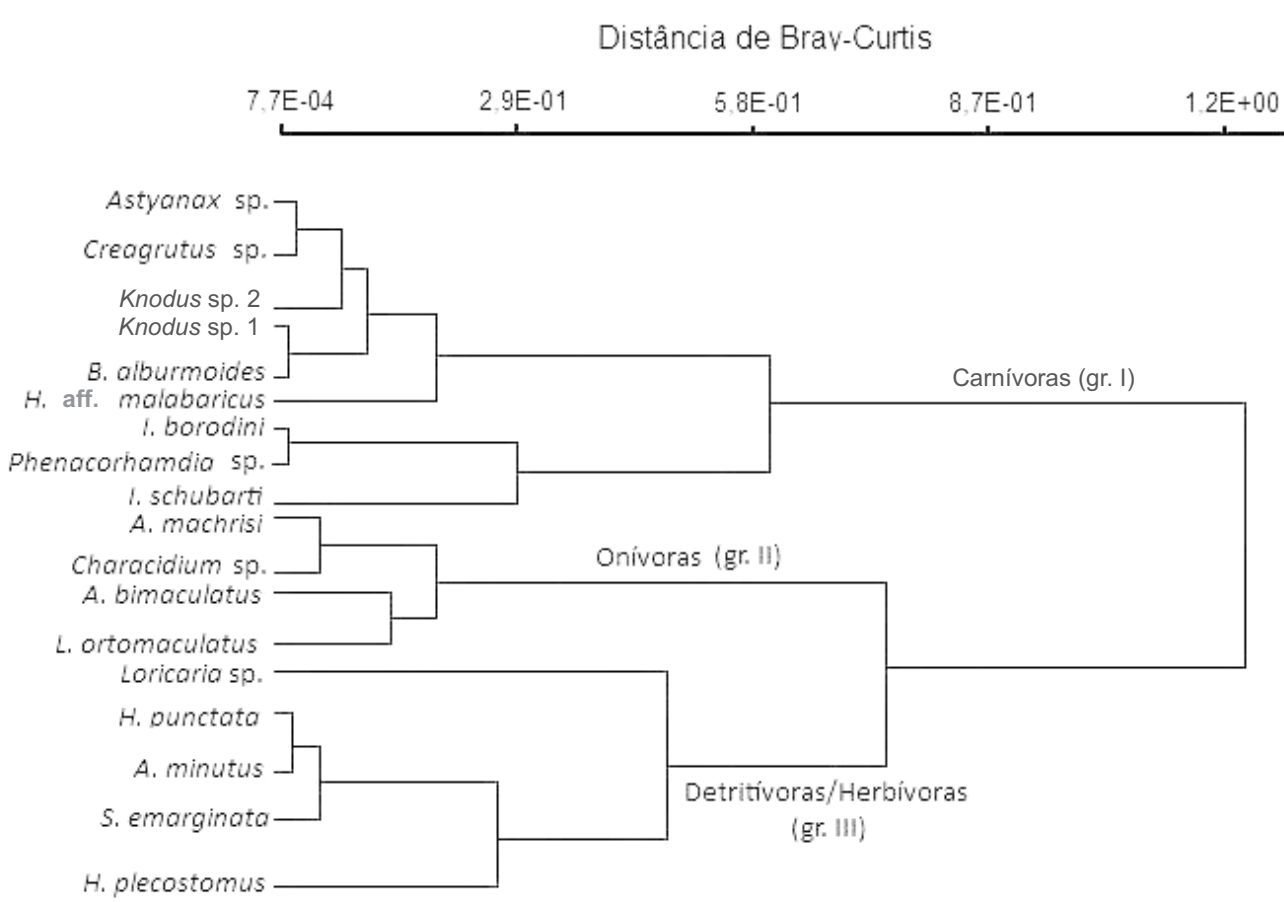

Figura 2. Dendrograma de similaridade baseado na composição da dieta das dezoito espécies de peixes analisadas no córrego Cavalo, GO, Brasil. 
daquelas com cabeça e olhos relativamente menores (Anostomidae, Parodontidae e Heptapteridae) (Fig. 3). A análise da co-variação entre as matrizes ecomorfológica e trófica evidenciou co-variação significativa (Mantel $\mathrm{t}=0,376 ; \mathrm{p}<0,001)$, indicando que os padrões morfológicos são relacionados ao hábito alimentar das espécies estudadas. Além disso, as comparações de Mantel para as matrizes trófica e ecomorfológica, contra a matriz taxonômica, revelou correlação significativa apenas para a primeira $(\mathrm{t}=8,38 ; \mathrm{r}=0,66 ; \mathrm{p}<0,001) \mathrm{e}$ ausência de correlação com a segunda $(\mathrm{t}=-0,98 ; \mathrm{r}=-0,07$; $\mathrm{p}=0,33)$.

Tabela III. Valores da correlação dos seis atributos ecomorfológicos nos dois eixos, com o primeiro eixo representando o componente principal 1 (PC1) e o segundo eixo representando o componente principal 2 (PC2), calculados para as dezoito espécies do córrego Cavalo, GO, Brasil (ARB, altura relativa da boca; ARC, altura relativa do corpo; $\mathrm{CRC}$, comprimento relativo da cabeça; CRI, comprimento relativo do intestino; DRO, diâmetro relativo do olho; OB, orientação da boca)

\begin{tabular}{|c|c|c|c|c|}
\hline \multirow[t]{2}{*}{ Atributos } & \multicolumn{2}{|c|}{ PC 1} & \multicolumn{2}{|c|}{ PC 2} \\
\hline & \multicolumn{3}{|c|}{ "loadings" Correlações "loadings" } & Correlações \\
\hline DRO & 0,43 & 0,37 & 0,37 & 0,52 \\
\hline CRI & $-2,14$ & $-0,48$ & 0,87 & 0,31 \\
\hline ARC & 0,54 & 0,44 & 0,29 & 0,38 \\
\hline $\mathrm{CRC}$ & $-0,22$ & $-0,33$ & 0,22 & 0,54 \\
\hline $\mathrm{ARB}$ & $-0,17$ & $-0,20$ & 0,22 & 0,43 \\
\hline $\mathrm{OB}$ & $-0,71$ & $-0,54$ & $-0,09$ & $-0,11$ \\
\hline Variância explicada (\%) & 51,5 & & 32,2 & \\
\hline Autovalores & 3,092 & & 1,935 & \\
\hline $\begin{array}{l}\text { Auto valores do } \\
\text { broken-stick }\end{array}$ & & 450 & & 450 \\
\hline
\end{tabular}

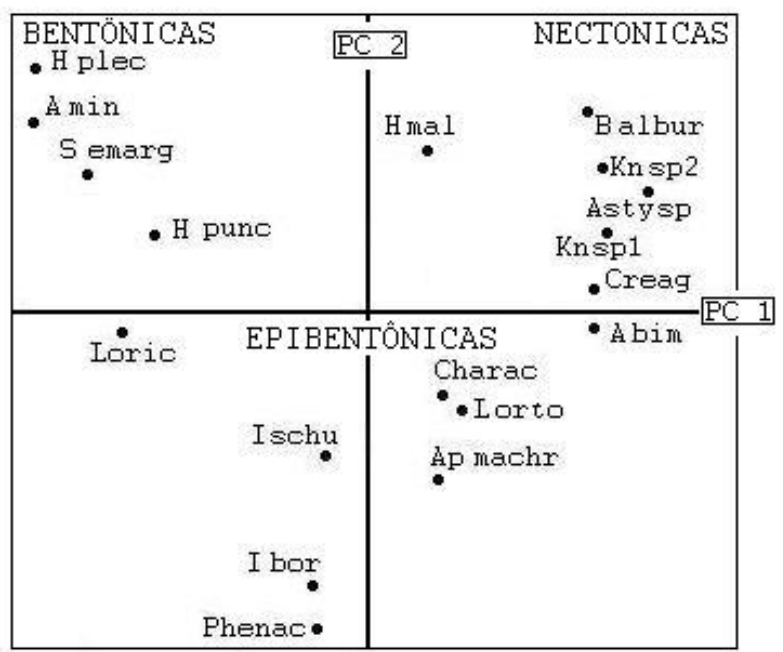

Figura 3. Análise de Componentes Principais baseado nos índices morfométricos das dezoito espécies estudadas no córrego Cavalo, GO, Brasil (A bim, Astyanax aff. bimaculatus; A min, Ancistrus minutus; Ap machr, Apareiodon machrisi; Asty sp, Astyanax sp.; B albur, Bryconops alburnoides; Charac, Characidium sp.; Creag, Creagrutus sp.; $\mathrm{H}$ mal, Hoplias aff. malabaricus; $\mathrm{H}$ plec, Hypostomus plecostomus; H punc, Harttia punctata; I bor, Imparfinis borodini; I schu, Imparfinis cf. schubarti; Kn sp1, Knodus sp.1.; Kn sp2, Knodus sp. 2.; Loric, Loricaria sp.; L orto, Leporinus ortomaculatus; Phenac, Phenacorhamdia SP; S emarg, Squaliforma emarginata).

\section{DISCUSSÃO}

O objetivo central dos estudos ecomorfológicos é a busca da compreensão das interações entre a morfologia dos organismos e a sua ecologia (Hugueny \& Poully, 1999). Os atributos ecomorfológicos devem refletir importantes aspectos da ecologia das espécies e servir de base para a compreensão/previsão dos aspectos que delineiam a história de vida das mesmas, tais como uso do hábitat e dos recursos tróficos (FERREIRA, 2007; Teixeira \& Bennemann, 2007). A despeito do incremento no número de trabalhos ecomorfológicos para peixes de riachos, observa-se que não há, ainda, um padrão consistente que permita afirmações conclusivas. Os resultados disponíveis são, muitas vezes, contraditórios, com alguns autores registrando forte correlação entre morfologia e ecologia (WINEMILLER et al., 1995), outros observando relação fraca ou parcial (Douglas \& Matthews, 1992; Casatti \& Castro, 2006) ou ausente (Felley, 1984; MotTA et al., 1995; Clifton \& MotTA, 1998; LABROPOULOU \& MARKAKIS, 1998) entre a morfologia e a ecologia das espécies. Neste contexto, vale ressaltar que os vários casos onde não foram registradas correlações positivas ocorreram em ambientes marinhos, possivelmente refletindo pressões seletivas distintas.

No presente estudo foi possível detectar padrões ecomorfológicos consistentes e amplamente fundamentados na relação entre os atributos morfológicos e o hábito alimentar das espécies estudadas. O agrupamento obtido a partir da ecomorfologia evidenciou diferenças estruturais relacionadas à altura do corpo, tamanho da cabeça e olhos e posição da boca, permitindo a distinção clara entre os Siluriformes e os Characiformes. A análise do espaço ecomorfológico obtido evidenciou diferenças estruturais fortemente associadas ao hábito alimentar das espécies em questão. Dentre os Siluriformes, por exemplo, Ancistrus minutus, Harttia punctata, Hypostomus plecostomus, Loricaria sp. e Squaliforma emarginata apresentaram atributos em comum (corpo deprimido dorso-ventralmente, boca em posição ventral, pedúnculo longo e nadadeiras peitorais e caudal de grande área) frequentemente relacionados a espécies bentônicas (GATZ, 1979; WATSON \& BALON, 1984; MatThews, 1998). O hábito alimentar detritívoro/herbívoro, fortemente associado às algas e itens do perifíton, corrobora o hábito bentófago dessas espécies.

Ainda dentre os Siluriformes, observa-se os Heptapteridae Imparfinis cf. schubarti, Imparfinis borodini e Phenacorhamdia sp., que se aproximaram no espaço ecomorfológico pela semelhança na forma do corpo, em especial no que se refere à posição subterminal da boca e ao corpo relativamente menos deprimido que as demais espécies de Siluriformes. Essas características justificam a proximidade morfológica dessas três espécies e podem explicar a semelhança no hábito alimentar, fortemente associado ao hábito semi-pelágico (epibentônico). De acordo com BALON et al. (1986) e FERREIRA (2007), esse tipo morfológico tem sido associado à capacidade de explorar recursos disponíveis em microambientes presentes entre pedras e pequenos espaços do substrato, em especial devido ao corpo 
maleável e pouco deprimido e à posição subterminal da boca, explicando a forte associação dessas espécies ao substrato (hábito bentônico/epibentônico). Por outro lado, a elevada ocorrência de itens de origem alóctone sugerem a exploração de recursos alimentares presentes na deriva, o que requer investidas na coluna d'água, fato que explicaria a possível associação com o hábito semipelágico, hábito também mencionado por CASATTI \& CASTRo (2006) para outras espécies de Heptapteridae.

Dentre os Characiformes, a exemplo do ocorrido com os Siluriformes, foi possível visualizar a segregação morfológica de dois grupos de espécies. Esse padrão de segregação caracterizou espécies de corpo alto e comprimido lateralmente e olhos grandes, com hábito alimentar orientado pela capacidade visual, e reuniu $B$. alburnoides, Astyanax sp., Creagrutus sp., as duas espécies de Knodus e H. aff. malabaricus. Essas espécies têm dieta baseada em itens de origem animal e apresentam comportamento tanto pelágico quanto epibentônico, se alimentando na coluna d'água e/ou no substrato, fato frequentemente registrado para as espécies desses gêneros (e.g. Uieda et al., 1997; CASTRO \& CASATTI, 1997). Destaca-se, nesse contexto, $H$. aff. malabaricus que, de acordo com os registros da literatura é uma espécie carnívora/piscívora (e.g. WinEMILLER, 1992; SILVA, 1993) e, no presente estudo, foi registrada se alimentando exclusivamente de larvas de insetos, o que pode ser explicado pelo fato de terem sido coletados apenas exemplares jovens (comprimento padrão entre 7,9 e 10,8 $\mathrm{cm}$, inferior ao da primeira maturação sexual, segundo MARQUES et al., 2007).

O segundo grupo de Characiformes foi formado pelos onívoros A. aff. bimaculatus, Characidium sp., Leporinus ortomaculatus e A. machrisi. Esse grupo reuniu espécies que se distinguiram pela falta de especialização do hábito alimentar, fato já mencionado por HoRn (1998). De fato, algumas dessas espécies têm sido classificadas em diferentes guildas tróficas de acordo com o ambiente em que se encontram. Por exemplo, espécies de Apareiodon foram classificadas como detritívoras e/ou herbívoras-perifitívoras em diversos estudos em outros sistemas fluviais (e.g. HöFLING et al., 2001; SAntin et al., 2004; CASATti \& CASTRO, 2006; Teixeira \& Bennemann, 2007). As espécies de Characidium têm sido classificadas como invertívoras (e.g. BARRETo \& ARANHA, 2006).

Os padrões de segregação evidenciados no presente estudo revelaram que a morfologia, além de estar fortemente associada ao hábito alimentar, reflete aspectos de uso do hábitat. Por exemplo, o grupo que reuniu os onívoros da amostra apresentou espécies de história evolutiva distinta, bem representada por diferentes gêneros e famílias. Conforme esperado, a variabilidade morfotípica das estruturas alimentares, tais como posição da boca e tamanho relativo do intestino, reflete o hábito alimentar generalista e flexível. Ainda nesse contexto, o grupo que reuniu as espécies de hábito carnívoro foi composto por espécies de Characiformes e Siluriformes (Heptapteridae, Characidae e Erythrinidae), de acordo com a revisão filogenética apresentada por MALABARBA et al. (1998) e ReIs et al. (2003), evidenciando um claro caso de convergência. As espécies reunidas nesse grupo têm posição filogenética relativamente bem definida e várias não compartilham ancestrais comuns, porém apresentaram dieta muito semelhante a despeito de, em alguns casos, utilizarem táticas de forrageamento distintas (para maiores detalhes ver CASATTI \& CASTRO $(1998,2006)$ ). De fato a correlação positiva entre as matrizes de alimentação e taxonômica reforçam essa proposta.

Evidências de casos de convergência e de divergência adaptativa foram igualmente registrados no presente estudo. Por exemplo, a presença das duas espécies de Astyanax ocorrendo em guildas distintas, assim como a segregação de Astyanax e Bryconops no espaço tanto ecomorfológico como no espaço trófico, seriam exemplos de divergência adaptativa, no caso de que a monofilia dos gêneros venha a ser reconhecida (e.g. Weitzman \& Malabarba, 1998; CASATti \& Castro, 2006). Segundo Casatti \& Castro (2006) o reconhecimento da monofilia desses gêneros seria fundamental para evidenciar um caso onde houve predomínio dos requerimentos ecológicos sobre os padrões filogenéticos, reforçando, assim, a evidência de que a evolução dos padrões filogenéticos/morfológicos podem ser fortemente influenciados pelos atributos ambientais.

Os grupos taxonômicos aqui estudados são os predominantes na ictiofauna de riachos da área de estudo (Miranda \& Mazzoni, 2003), além de serem os de maior representatividade na ictiofauna de sistemas fluviais sulamericanos (Lowe-McConnell, 1987; CASTRO, 1999). A análise ecomorfológica revelou-se uma ferramenta adequada na descrição de aspectos ecológicos dessas espécies, refletindo preferências alimentares e de uso do hábitat e pode ser usada como método preditivo da estrutura trófica da comunidade estudada.

Agradecimentos. Aos membros dos laboratórios de ecologia de peixes da UERJ e da UFRJ pelo auxílio no trabalho de campo e processamento de parte do material. A Ricardo IglesiasRios pelas críticas nas diversas etapas de desenvolvimento do trabalho. Esse trabalho foi realizado através do contrato Serra da Mesa Energia-Furnas / BioRio / UFRJ, convênio UERJ e foi beneficiado pelo CNPq através da concessão de bolsas individuais para RM- 301433/2007-0 e CFR- 434 140928/2005-7.

\section{REFERENCIAS BIBLIOGRÁFICAS}

Balon, E. K.; Crawford, S. S. \& Lelek, A. 1986. Fish communities of the upper Danube river (Germany, Austria) prior to the new Rhein-Main-Donau connection. Environmental Biology of Fish 15(4):243-271.

Barrella, W.; Beaumord, A. C. \& Petrere-Jr, M. 1994. Comparison between the fish communities of Manso river (MT) and Jacaré Pepira river (SP), Brazil. Acta Biologica Venezuelana 15(2):1-15.

Barreto, A. P. \& Aranha, J. M. 2006. Alimentação de quatro espécies de Characiformes de um riacho da Floresta Atlântica, Guaraqueçaba, Paraná, Brasil. Revista Brasileira de Zoologia 23(3):779-788.

Beaumord, A. C. \& Petrere-Jr, M. 1994. Fish communities of Manso river, Chapada dos Guimarães, MT, Brasil. Acta Biologica Venezuelana 15(2):21-35.

Casatti, L. \& Castro, R. M. C. 1998. A fish community of the São Francisco river headwaters riffles, southeastern Brazil. Ichthyological Exploration of Freshwaters 9(3):229-242. 2006. Testing the ecomorphological hypothesis in a headwater riffles fish assemblage of the rio São Francisco, southeastern Brazil. Neotropical Ichthyology 4(2):203-214. 
Castro, R. C. M. 1999. Evolução da ictiofauna de riachos Sulamericanos: Padrões gerais e possíveis processos causais. In: Caramaschi, E. P.; Mazzoni, R. \& Peres-Neto, P. R. eds. Ecologia de peixes de riacho. Série Oecologia Brasiliensis. Rio de Janeiro, Computer \& Publish. v.6, p.139-156.

Castro, R. C. M. \& Casatti, L. 1997. The fish fauna from a small forest stream of the upper Paraná river basin, Southeastern Brazil. Ichthyological Exploration of Freshwater 7(4):337-352.

Clifton, K. B. \& Motta, J. P. 1998. Feeding morphology, diet and ecomorphological relationships among five Caribbean labrids (Teleostei, Labridae). Copeia 1998(4):953-966.

Cunico, A. M. \& Agostinho, A. A. 2006. Morphological patterns of fish and their relationships with reservoirs hydrodynamics. Brazilian Archives of Biology and Technology 49(1): 125-134

Douglas, M. E. \& Endler, J. A. 1982. Quantitative matrix comparisons in ecological and evolutionary investigations. Journal of Theoretical Ecology 99:777-795.

Douglas, M. E. \& Matthews, W. J. 1992. Does morphology predicts ecology? Hypothesis testing within a fish assemblage. Oikos 65:213-224

Felley, J. D. 1984. Multivariate identification of morphological - environmental relationships within the Cyprinidae (Pisces). Copeia 1984(2):442-455.

Ferreira, K. M. 2007. Biology and ecomorphology of stream fishes from the river Mogi-Guaçu basin, Spoutheastern Brazil. Neotropical Ichthyology 5(3):311-326.

Freire, A. G. \& Agostinho, A. A. 2001. Ecomorfologia de oito espécies dominantes da ictiofauna do reservatório de Itaipu (Paraná/ Brasil). Acta Limnologica Brasiliensia 13(1):1-9.

GAtZ, A. J. 1979. Ecological morphology of freshwater stream fishes. Tulane Studies in Zoology and Botany 21(2):91-124.

Höfling, J. C.; Ferreira, L. I.; Ribeiro-Neto, F. B.; Santos, M. V. S. \& Martins, T. 2001. Alimentação de Apareiodon affinis e Geophagus brasiliensis na represa de Jaguarí-Jacareí, SP, Brasil. Bioikos 15(1):7-10.

Horn, M. H. 1998. Feeding and digestion. In: Evans, D. H. ed. The physiology of fishes. Boca Raton, CRC. p.43-64.

Hugueny, B. \& Pouilly, M. 1999. Morphological correlates of diet in an assemblage of West African freshwater fishes. Journal of Fish Biology 54(6):1310-1325.

Hyslop, E. J. 1980. Stomach contents analysis - a review of methods and their application. Journal of Fish Biology 17(4):411-429.

Labropoulou, M. \& Markakis, G. 1998. Morphological-dietary relationships within two assemblagens of marine demersal fishes. Environmental Biology of Fishes 51(3):309-319.

Lowe-McConell, R. H. 1987. Ecological Studies in Tropical Fish Communities. Cambridge, Cambridge University. 382p.

Mahon, R. 1984. Divergent structure in fish taxocenes of north temperate streams. Canadian Journal of Fisheries and Aquatic Sciences 41:330-350.

Malabarba, L. R.; Reis, R. E.; Vari, R. P.; Lucena, Z. M. S. \& Lucena, C. A. S. 1998. Phylogeny and classification of neotropical fishes. Porto Alegre, Edipucrs. 603p.

Marques, C. S.; Braun, A. S. \& Fontoura, N. F. 2007. Estimativa de tamanho de primeira maturação a partir de dados de IGS: Oligosarcus jenynsii, Oligosarcus robustus, Hoplias malabaricus, Cyphocharax voga, Astyanax fasciatus (Characiformes), Parapimelodus nigribarbis, Pimelodus maculatus, Trachelyopterus lucenai, Hoplosternum littorale, Loricariichthys anus (Siluriformes) e Pachyurus bonariensis (Perciformes) no lago Guaíba e laguna dos Patos, RS. Biociências 15(2):230-256.

Matthews, W. J. 1998. Patterns in freshwater ecology. New York, Chapman \& Hall. 756p.

Mazzoni, R.; Fenerich-Verani, N. \& Caramaschi, E. P. 2000. Electrofishing as a sampling technique for coastal stream fish populations and communities in the southeast of Brazil Brazilian Journal of Biology 60(2):205-216.

McCune, B. \& Mefford, M. J. 1997. PC-ORD. Multivariate Analysis of Ecological Data. Version 3,0. MjM Software Oregon, Gleneden Beach.

Merrit, R. W. \& Cummins, K. W. 1984. An introduction to the aquatic insects of North America. Lowa, Kendall/Hunt. 722p.

Miranda, J. C. \& Mazzoni, R. 2003. Composição da ictiofauna de três riachos do alto rio Tocantins, GO. Biota Neotropica 3(1): $1-11$.

Motta, P. J.; Clifton, K. L. B.; Hernandez, P. \& Eggold, B. T. 1995. Ecomorphology correlates in ten species of subtropical seagrass fishes: diet and microhabitat utilization. Environmental Biology of Fishes 44(1-3):37-60.

Pérez, G. R. 1988. Guia para el estúdio de los macroinvertebrados acuáticos del Departamento de Antioquia. Bogotá, Universidad de Antioquia. 215p.

PIET, G. J. 1998. Ecomorphology of a size-structured tropical freshwater fish community. Environmental Biology of Fishes 51(1):67-86.

Poullly, M.; Lino, F.; Bretenoux, J. G. \& Rosales, C. 2003. Dietarymorphological relationships in a fish assemblage of the Bolivian Amazonian floodplain. Journal of Fish Biology 62(6): 1137-1158.

Reis, R. E.; Kullander, S. O. \& Ferraris-Jr, C. J. 2003. Check list of the freshwater fishes of South and Central America. Porto Alegre, Edipucrs. 729p.

Rincón, P. A. 1999. Uso do micro-hábitat em peixes de riacho: Métodos e perspectivas. In: Caramaschi, E. P.; Mazzoni, R. \& Peres-Neto, P. R. eds. Ecologia de peixes de riacho. Série Oecologia Brasiliensis. Rio de Janeiro, Computer \& Publish. v.6, p.23-90

Santin, M.; Bialetzki, A. \& Nakatani, N. 2004. Mudanças ontogênicas no trato digestório e dieta de Apareiodon affinis (Steindachner, 1879) (Osteichthyes, Parodontidae). Acta Scientiarum Biological Sciences 26(3):291-298.

Silva, C. P. D. 1993. Alimentação e distribuição especial de algumas espécies de peixes do igarapé do Candirú, Amazonas, Brasil. Acta Amazonica 23(2):271-285.

Stevens, R. \& Willig, M. R. 2000. Community structure, abundance, and morphology. Oikos 88(1):48-56.

Teixeira, I. \& Bennemann, S. T. 2007. Ecomorfologia refletindo a dieta dos peixes em um reservatório no sul do Brasil. Biota Neotropica 7(2):67-77.

Uieda, V. S.; Buzzato, P. \& Kikuchi, R. M. 1997. Partilha de recursos alimentares em peixes em um riacho de serra do sudeste do Brasil. Anais da Academia Brasileira de Ciências 69(2):243-252.

WATson, D. J. \& BALON, E. K. 1984. Ecomorphological analysis of fish taxocenes in rainforest stream of northern Borneo. Journal of Fish Biology 25(3):371-384.

Weitzman, S. H. \& Malabarba, L. R. 1998. Perspectives about the phylogeny and classifications of the Characidae (Teleostei: Characiformes). In: Malabarba, L. R.; Reis, R. E.; Vari, R. P.; Lucena, Z. M. S. \& Lucena, C. A. S. eds. Phylogeny and classification of Neotropical fishes. Porto Alegre, Edipucrs. p.161-170.

WiKRAMANAYAKE, E. D. 1990. Ecomorphology and biogeography of a tropical stream fish assemblage: evolution of assemblage structure. Ecology 71(5):1756-1764.

Winemiller, K. O. 1992. Ecomorphology of freshwater fishes. Research Exploration 8(3):308-327.

Winemiller, K. O.; Kelso-Winemiller, L. C. \& Brenkert, A. L. 1995. Ecomorphological diversification and convergence in fluvial cichlid fishes. Environmental Biology of Fishes 44(1-3):235-261.

XIE, S.; CUI, Y. \& Li, Z. 2001. Dietary-morphological relationships of fishes in Liangzi Lake, China. Journal of Fish Biology 58(6): 1714-1729.

Recebido em outubro de 2009. Aceito em abril de 2010. ISSN 0073-4721

Artigo disponível em: www.scielo.br/isz 